\title{
Satellite selection in the context of an operational GBAS
}

\author{
Daniel Gerbeth(1) | Maria Caamano | Mihaela-Simona Circiu | Michael Felux(i)
}

German Aerospace Center (DLR), Oberpfaffenhofen 82234, Wessling, Germany

\section{Correspondence}

Daniel Gerbeth, German Aerospace

Center (DLR), Germany.

Email: daniel.gerbeth@dlr.de

\begin{abstract}
When incorporating multiple constellations into future ground based augmentation systems (GBAS), a problem with limited VDB (VHF data broadcast) capacity might arise. Furthermore, the number of airborne receiver tracking channels could be insufficient to use all visible satellites. One way to cope with these issues is to perform a satellite selection to limit the number of used satellites with minor impact on performance. This paper investigates different factors that constrain the approach of simply selecting "the best set in every epoch" and shows how to overcome some limitations. These constraints include limitations in satellite visibility, loss of satellites during approach (i.e. in curves), and convergence times in the airborne processing until satellites are usable.

Various protection level simulations are performed to show the influence of the named factors on the nominal performance. Taking into account all these contextual influences, results show satellite selection is still applicable in GBAS ground stations.
\end{abstract}

\section{1 | INTRODUCTION}

The ground based augmentation system (GBAS) in its currently commercially available version provides precision approach guidance for CAT I approaches. In GBAS terminology, this is called GBAS approach service type (GAST) C. In the near future, the development and standardization of a CAT II/III capable system (the so-called GAST D) will be completed. Both of these service types, however, are expected to have very limited availability in regions with active ionospheric conditions. Therefore, a future service type (GAST F) using two frequencies and multiple constellations of global navigation satellite systems (GNSS) is currently under development. One of the main constraining factors in the definition of a new multi-frequency multi-constellation (MFMC) concept is the limited capacity of the VDB (VHF data broadcast) link which is used to provide corrections and integrity parameters to landing aircraft.

In order to be backwards compatible with GAST C (and potentially also GAST D) users, the current GPS L1 cor- rections and integrity parameters still have to be broadcast by a GAST F ground station. One possibility to overcome this capacity problem is to reduce the update rate for the corrections of further frequencies and constellations. To fulfill the time to alert requirements, additional integrity messages have to be broadcast with a higher update rate, and the integrity concept would have to be revised in order to ensure that with the lower correction update rate, all requirements can still be met. ${ }^{1}$

The work in this paper is taking an alternative approach: For GAST F, the ground station broadcasts only additional corrections and integrity parameters for a thoroughly selected subset of all visible satellites. The corrections transmitted for GAST C and D services are left unchanged and are kept for legacy users. This allows maintaining the current update rate while still benefiting from the better geometric diversity of multiple constellations as well as a second frequency.

Satellite selection in general was a topic of several papers in the past, providing different algorithms to perform the task. The common goal is in general to find a sub- 
set of all satellites currently in view which provides the best (whereby best depends on the application) navigation performance under given side conditions. Simple and computationally efficient approaches using only satellite elevation and azimuth were presented by Zhang, ${ }^{2}$ Song, ${ }^{3}$ and Park. ${ }^{4}$ Also, the widespread approach of selecting the highest satellites, assuming the best signal quality with highest elevation, falls into this category. Another group of algorithms tries to maximize polyhedron volumes or matrix determinants ${ }^{5-7}$ as they correlate well with the geometric dilution of precision (GDOP) of a set. Both of these strategies suffer from the missing possibility to take into account any satellite weighting (eg, due to signal quality) and focus on 3D positioning, making them less suitable in our context. Phatak presented a method ${ }^{8}$ which allows efficient exchange of single satellites in a set which we utilize in our approach as well. Beyond that, approaches applying genetic algorithms ${ }^{9}$ or artificial neural networks ${ }^{10}$ were presented earlier.

Aside from the incorporated algorithm itself, herein we specifically focus on the influence of operational constraints on the use of satellite selection in GBAS. These constraints come from different factors, mainly satellite visibility (above the elevation limit) and filter convergence times. When a GBAS ground station makes a selection of satellites to broadcast corrections for, these factors should be considered instead of just selecting the satellites that lead to the best performance at a given epoch right at the ground station's location. While the topic of satellite selection in general has been largely covered very recently, ${ }^{11-14}$ this work is not limited to a specific selection algorithm but valid for any kind of selection. The objective is rather to show that satellite selection in GBAS is not largely affected by operational implications that cannot be avoided. Three main aspects covering this topic are primarily addressed in this paper:

- Optimizing the selection on ground for the whole GBAS service volume and considering convergence times

- Considering satellite-out cases in the selection process to avoid single performance-crucial satellites

- Strategies for airborne receivers not capable of tracking all satellites in view or with limited processing power

\section{2 | METHODS}

\section{1 | General assumptions}

First of all, we want to make some general statements in terms of the simulations and studied aspects in this paper. One important fact to mention again is that the studies we are going to present are not specific to a single selection method. Instead, the results and implications apply to any kind of satellite selection in GBAS. As the used algorithm in this work, we utilize our previously presented optimization method ${ }^{12}$ with slight modifications on the selection criteria in line with Walter et al. ${ }^{11}$ Using an exhaustive search instead could change some of the results slightly, but as Gerbeth et al ${ }^{12}$ showed the heuristic algorithm to differ by well below $1 \%$ on average, we decided on a heuristic approach for the reason of inapplicable computation times otherwise.

The general idea of the heuristic selection is to use a measure based on all satellites currently in view which allows judging of the probability of a satellite to be part of a best-n set. This measure to perform the initial selection is given as

$$
s_{\text {measure }, i}=\frac{\left(s_{3, i}+\tan \left(\theta_{G P A}\right) \cdot s_{1, i}\right)^{2}}{p_{i, i}}
$$

with

$$
\mathbf{S}=\left(\mathbf{G}^{T} \cdot \mathbf{W} \cdot \mathbf{G}\right)^{-1} \mathbf{G}^{T} \cdot \mathbf{W}
$$

and

$$
\mathbf{P}=\mathbf{W}-\mathbf{W} \cdot \mathbf{G} \cdot \mathbf{S}
$$

where $\mathbf{G}$ is the satellite geometry matrix in ENU (East North Up coordinates) and $\mathbf{W}$ is the weighting matrix. ${ }^{15}$ Lowercase variables $\left(s_{i, j}\right)$ refer to the element in row $i$ and column $j$ of the respective matrix (uppercase). The angle $\theta_{G P A}$ describes the glide slope inclination of the current approach path, typically about 3 degrees. While our previous work used $s_{\text {vert }}$ as criteria to estimate the "value" of a satellite within a limited subset, the updated measure can be considered a weighted $s_{\text {vert }}$.

Using this measure, an initial set of satellites is selected. The initial selection simply consists of the $n$ satellites with the highest measure according to Equation 1. This first set is iteratively optimized in a "greedy exchange" way. A detailed overview of this optimization can be found in Gerbeth et al. ${ }^{12}$

To judge and compare the performance in the following studies, we mostly utilize vertical protection levels (VPLs), overbounds of the remaining differential position errors, as they give a good indication of the nominal performance to expect. The focus is on the vertical domain here because the vertical protection is both harder to fulfill and more critical during final approach and landing. Details on the computation of the protection levels can be found in RTCA DO-253D. ${ }^{15}$ As the absolute value of the achieved protection levels is also not of major interest in this study, but instead the relative changes due to different modifications on the selection, we will not go into details here and refer to the standards or our previous works instead. ${ }^{12,16}$ 


\subsection{Service volume and convergence time considerations}

\subsection{1 | Limiting the exchange rate of satellites over time}

A first aspect we want to consider in terms of optimizing the selection on the ground is the rate at which satellites in the selected set are exchanged. This relates partially to the airborne filter convergence times. But apart from filter convergence, additional operational requirements apply before a satellite can be incorporated in the GBAS solution. In GAST D, especially, a 200-second delay (see RTCA DO-253D, ${ }^{15}$ Section 2.3.9.7) is required from the DSIGMA monitoring when the user is within the precise approach region (PAR). Depending on future monitoring and requirements in GAST F, the most stringent delay should be considered when the ground selection is performed.

Here, we assume an airborne user without capability of tracking all satellites in view. Any change in the set of received corrections (ie, the ground selection) would require the airborne user to replace the satellite. When the newly used satellite was not tracked or incorporated in the GBAS processing before, it requires some time until it is usable. Until then, the aircraft would be limited to the remaining satellites. In the rare case of multiple satellites exchanged within a short time, the airborne user could experience a significantly degraded performance with the previous satellites no longer (no corrections) and the new satellites not yet (not converged) usable.

An option to overcome this issue is to limit the number of exchanged satellites to one per time-step and additionally introduce a timeout $\tau_{\text {exchange }}$ between changes. This timeout should be based on the airborne convergence time and applicable delays (until a satellite can be used) due to airborne monitors. After an exchange, the ground selection should wait for at least $\tau_{\text {exchange }}$ seconds before the next satellite is exchanged. This could sometimes mean that the selection is no longer optimal during an improving exchange when the timeout had not yet passed. Only in the case where an airborne user can track and use all satellites in view could a change in the set of received corrections be incorporated without any delay.

\subsection{2 | Handling of rising and setting satellites}

The setting of satellites below the elevation limit, which we want to consider as a second aspect, is related to the satellite visibility regions. When a satellite is newly included into a selection, it might take the airborne processing some time to actually use it, depending, ie, on the number of receiver channels. To tackle this problem, we introduce a minimum time the satellite needs to stay above this limit to be included in the selected set. The minimum time is based on the convergence time of the airborne filters. Any satellites setting within a time smaller than the actual filter convergence time would not grant any use for airborne users. Therefore, minimum remaining usability times of at least $2 \tau$ can be considered reasonable (where $\tau$ is the airborne convergence time, ie, the time until the satellite would actually be used).

Satellites that set within a shorter time span are not included into the selection. This does not affect satellites already present in the currently broadcast set. These stay within the selection as long as they are above the elevation limit or until replaced by another satellite for performance reasons (ie, smaller protection level).

\subsection{3 | Satellite visibility within the service volume}

The last aspect we want to consider in this section is the satellite visibility within the service volume in general. When performing any satellite selection in the GBAS ground station, it has to be taken into account that very low elevation satellites are not necessarily usable for all airborne users. Depending on the distance to the ground station and the altitude of the aircraft, a satellite might be still (or already) below the elevation mask and therefore unusable. Broadcasting corrections for these satellites might be of no use for at least part of the users and therefore degrade the average performance. It is consequently desirable to take the influence of satellite visibility into account when performing the selection on the ground.

We will compare two different methods for how this can be achieved. The first simple approach is to raise the elevation limit for satellites taken into account during selection. By raising the elevation limit by about one degree, it can be made certain that the selected satellites are visible (above the elevation limit) to all users within the service volume of $43 \mathrm{~km}$. This assumes the airborne users to be not more than $3000 \mathrm{~m}$ above the ground reference, as height difference is one of the two influencing factors on the local satellite elevation. When a larger radius than $43 \mathrm{~km}$ is supposed to be served, then this limit on the ground has to be adjusted accordingly, as the curvature of Earth accounts for the second influencing factor. While this approach is easy to implement and makes sure no corrections are sent that are "needless" for some users, it also comes with an expected loss of performance. From a geometric point of view, low elevation satellites provide a large contribution to the achieved performance. Removing the lowest one or more degrees of elevation over all azimuths can therefore influence the protection levels significantly in some situations. 
To overcome this, the second approach is more complex. The idea is to sample the service volume at relevant locations (marked as red crosses in Figure 1) and perform independent selections. These locations we use are defined by intersections between edges where satellite visibility regions start/end (gray intersecting lines) and additionally the edge of the service volume (black circle). Afterwards, the performance of the different sets is computed throughout the service volume and the best of these sets is finally broadcast. This procedure is by far more complex and requires a significantly higher processing power, but also is more flexible and offers tuning opportunities. One option, for example, is to optimize the selection only for the (known) approach routes, ignoring areas where no arriving flights are passing normally. This can offer quite some potential as can be seen from the approaches towards Frankfurt Airport in Figure 2. The major part of the GBAS service volume is actually never used. Whether a satellite is visible in these regions or not is consequently not relevant, easing the constraints which satellite corrections to broadcast.

\subsection{Consideration of satellite-out cases}

In this section, we want to study the influence of satellite outages on the selection. The question is especially: Can we make our selection more robust against sudden satellite drops by giving up some nominal performance? These outages are not so rare, when considering curves during the approach. Bank angles up to 30 degrees make these outages especially immanent to low elevation satellites, when the wings or fuselage cut the line of sight.

This probability of lost satellites during an approach can be incorporated into the selection process by replacing the common VPL as measure to judge the quality of a set. Instead, an adjusted "protection level" is computed

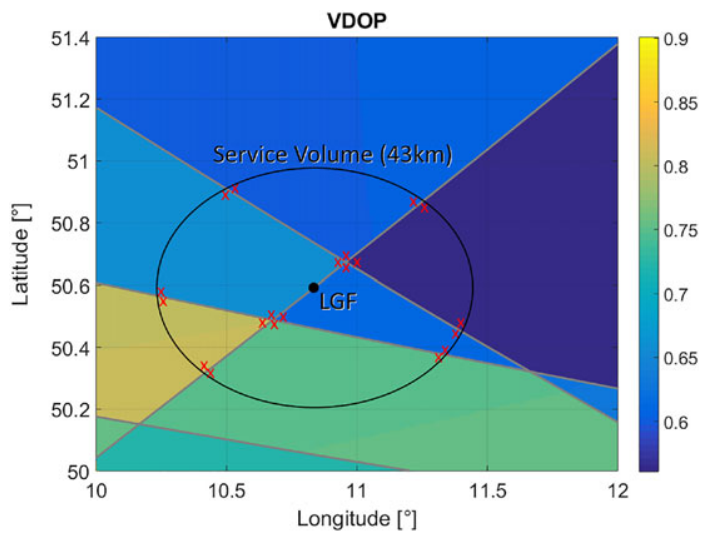

FIGURE 1 Example for satellite visibility across GBAS service volume [Color figure can be viewed at wileyonlinelibrary.com and www.ion.org]

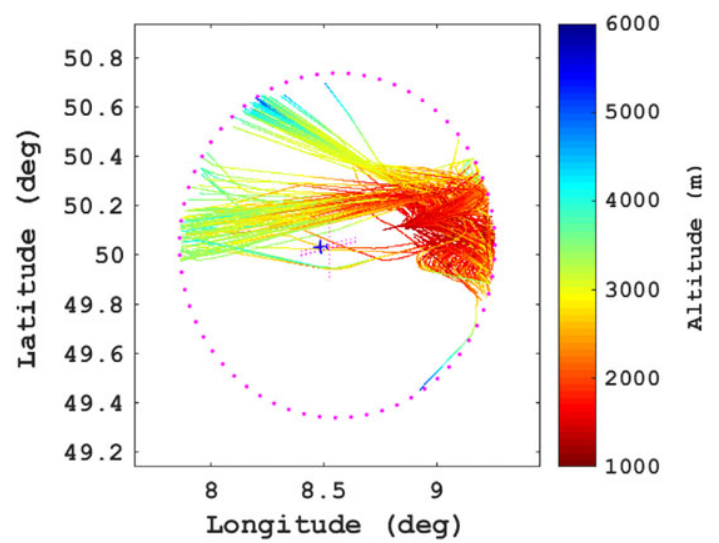

FIGURE 2 Example plot of 1 day of approaches towards Frankfurt Airport, taken from Kumar et $\mathrm{al}^{17}$ [Color figure can be viewed at wileyonlinelibrary.com and www.ion.org]

by combining the protection levels of possible out-cases weighted by their probability of occurrence. The adjusted protection level for judging the sets is given as

$$
\begin{aligned}
V P L_{\text {adjusted }}= & p_{e, 0} \cdot V P L_{\text {full }}+p_{e, 1} \cdot V P L_{1}+p_{e, 2} \cdot V P L_{2} \\
& +\ldots+p_{e, 1} \cdot p_{e, 2} \cdot V P L_{1,2}+\ldots \ldots
\end{aligned}
$$

with $p_{e, i}$ as the outage probability of satellite $i$ and $V P L_{i}$ the protection level with satellite $i$ removed. We assume $p_{e, 0}$ as the probability of no satellite outage, ie, complementary probability to all out-cases considered.

We use the probability of a satellite outage following a piecewise linear function over elevation $e l$ given by:

$$
p_{e}(e l)=\left\{\begin{array}{lr}
0.20, & 0<e l<20 \\
0.20 \cdot(40-e l) / 20, & 20 \leq e l<40 \\
0, & 40 \leq e l \leq 90
\end{array}\right.
$$

Thus, we assume a constant maximum outage probability of $20 \%$ up to 20 degrees of elevation. Afterwards, from 20 to 40 degrees of satellite elevation, the probability drops linearly towards zero. This can be seen as a conservative overbound of expected loss probability during curvy approaches and was modeled after DLR flight trial data. The general selection procedure as we described before stays the same. A starting set is selected based on the $s_{\text {measure }}$ and then gradually optimized by single exchanges. Instead of common $V P L$, the new $V P L_{\text {adjusted }}$ is minimized.

\section{4 | Airborne selection strategies}

In this section, we must distinguish between different scenarios. One is dedicated to stand-alone positioning, ie, before transitioning into the GBAS service. This is also relevant in the case of, for example, unmanned aerial systems (UAS) with limited battery life and strong demand on (power) efficient positioning. The second topic regards 


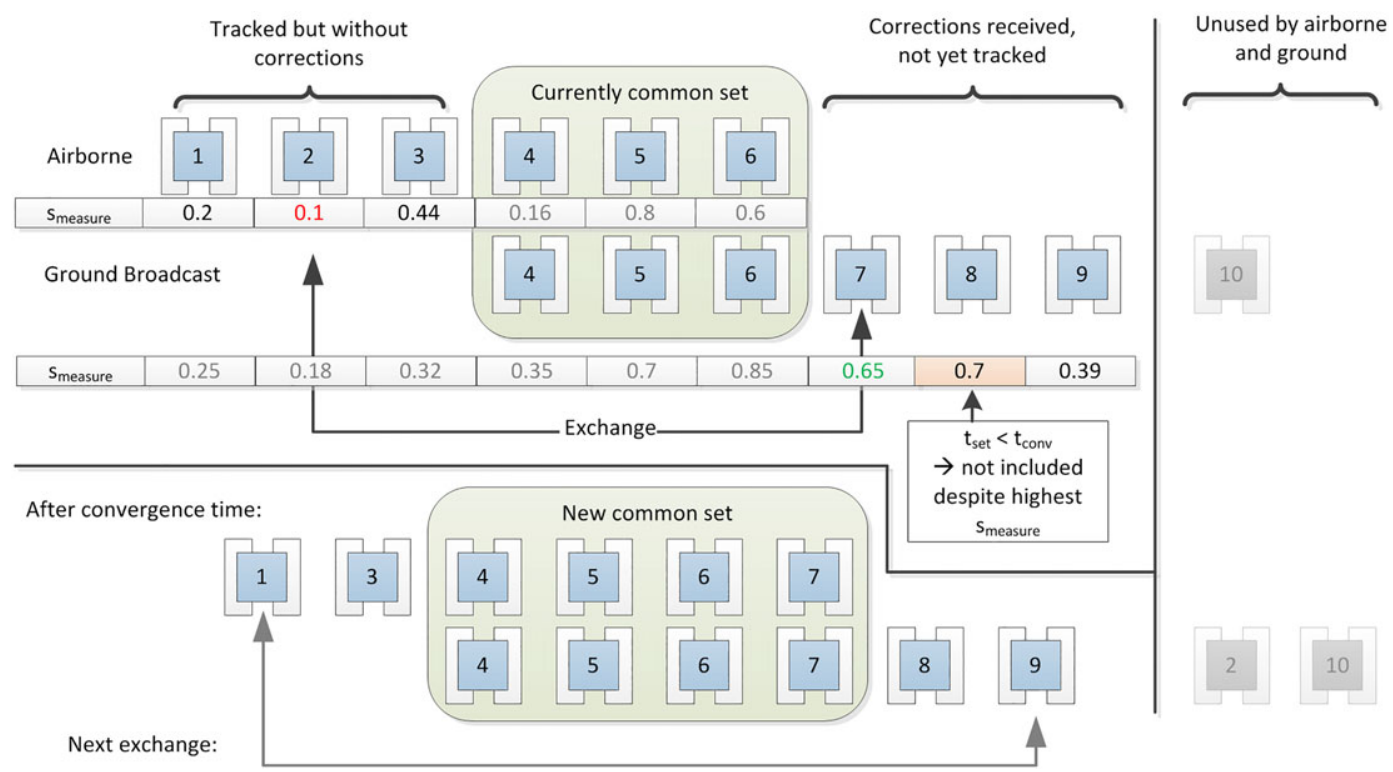

FIGURE 3 Sketch of the airborne satellite exchange scheme for transition into GBAS. In the example, PRN 2 is exchanged with PRN 7. PRN 8 is ignored due to imminent set below the elevation mask. Instead of using the $s_{\text {measure }}$ for determining the exchanges, an exhaustive (brute force) search could be performed for optimal results [Color figure can be viewed at wileyonlinelibrary.com and www.ion.org]

the actual transition from a not differentially corrected position (using, eg, SBAS or ARAIM) to the use of GBAS. Considering an airborne user already using the selection strategy en-route, eg, when using SBAS, the used satellites are likely better aligned with the expected situation at the GBAS station, making the transition more seamless. In this case, it is also beneficial to have similar algorithms running on both air and ground, to maximize interoperability while limiting certification effort and thereby costs.

\subsection{1 | Stand-alone users in continuous operation}

In terms of continuous operation of stand-alone users, an approach was presented quite recently from Swaszek et al. ${ }^{13}$ Similarly in Gerbeth et al, ${ }^{12}$ we already used the previously selected set as one of our starting sets for the optimization problems in continuous operation. If necessary, this can be even further simplified by performing a search for the best subset only in case of trigger events. This reduces the required computations drastically while having a minor impact on the achieved performance.

We can identify three simple trigger events which allow efficient scheduling of new satellite selections during continuous operation:

- Rise of a new satellite

- Setting of a currently used satellite

- Time since the last set search/optimization
All of them are quite obvious, as the slowly evolving satellite geometry changes mostly when new satellites become usable or previous ones drop out. An additional timed trigger, eg, every 5 to 10 minutes, allows "coasting" through periods without set or rise events. This could also be triggered whenever processing power is "free" in performance critical UAS applications, enabling a flexible, still optimal performance.

\subsection{2 | Transition to GBAS}

In terms of a user approaching a GBAS station to perform a landing, it is necessary to align the tracked and used (in the GBAS processing) satellites with the ones provided with corrections from the ground. If the airborne receiver is capable of tracking all satellites in view (of the supported constellations), this is trivial. The GBAS processing can just utilize the measurements from all corrected satellites in this case.

Otherwise, there needs to be a procedure to exchange satellites currently tracked with the satellites provided with corrections from the ground station. The algorithm we propose for this is fairly straightforward. To limit the drop in performance due to a sudden exchange of many satellites (which can happen in a multi-constellation scenario), they should be exchanged one by one. The timeout between the exchanges can be equal to the filter convergence times, ie, 30 or 100 seconds in the current GBAS. Exchanges should be performed in a way that the least influential satellite in the tracked set is exchanged with the 
most beneficial from the satellites with broadcast corrections. One simple measure that allows judging the influence on the performance without any brute force iterations is the one given in Equation 1. From the currently tracked satellites that are not in the broadcast, the satellite with the smallest $s_{\text {measure }}$ (ie, a small contribution to the navigation performance) would be replaced first.

The replacement would be the satellite with the highest $s_{\text {measure }}$ of the remaining satellites. In this case, the measure would be calculated for the all-in-view solution, using almanac data to compute azimuth and elevation of the satellites where necessary. One restriction has to be made here: satellites about to set below the horizon should be excluded from the procedure. Three cases can be distinguished based on the time until a satellite sets below the elevation mask:

- $t_{\text {set }} \lesssim t_{\text {convergence }}$ : The satellite sets before the airborne filters are even converged and is therefore ignored.

- $t_{\text {convergence }}<t_{\text {set }}<t_{\text {landing: }}$ : The satellite sets during the expected time until landing. It is therefore treated with lower priority.

- $t_{\text {set }}>t_{\text {landing }}$ : The satellite stays visible during approach and landing. These satellites are exchanged first.

The remaining time that satellites stay above the elevation mask, $t_{s e t}$, can be estimated using the ephemeris or almanac data. Filter convergence $t_{\text {convergence }}$ is known from the performed processing. For $t_{\text {landing }}$, estimations have to be made either based on the actual current approach route or, if not available, distance and speed(-profile) until touchdown. An illustration of the exchange scheme is given in Figure 3.

\section{3 | RESULTS AND DISCUSSION}

As mentioned earlier, the protection levels we use here as comparative measure are computed according to RTCA DO-253D. ${ }^{15}$ As assumptions for $\sigma_{\text {air }}$ and $\sigma_{\text {ground }}$, we use models developed at DLR and previously published in an open access article. ${ }^{16}$

Some additional general simulation parameters are collected in the following list:

- Current GPS (30 sat.) and nominal future Galileo (27 sat.) almanacs for satellite orbits

- Aircraft velocity $70 \mathrm{~m} / \mathrm{s}$, except for the final simulation where a speed profile is used

- $\sigma_{\text {air }}$ and $\sigma_{\text {ground }}$ as in Gerbeth et al, ${ }^{16} 100$-second models for both GPS L1 and Galileo E1

- GBAS ground station broadcasts corrections for 12 satellites from dual constellation

- Airborne receiver tracks 12 satellites
- Locations at low $\left(0^{\circ}\right)$, medium $\left(55^{\circ}\right)$, and high latitudes $\left(75^{\circ}\right)$ and $10^{\circ}$ longitude

- We only compare heuristic selections: the baseline "best sets" are always also heuristically found and not by exhaustive search

- We only consider H0 protections levels, not ephemeris or H1 PL (see RTCA DO-253D ${ }^{15}$ for details)

- The protection levels can be considered as GAST C protection levels without sigma-inflation or GAST D protection levels without $D_{v} / D_{l}$ terms

- No airborne geometry screening is performed, but due to the multi-constellation setup and 12 used satellites, $s_{\text {vert }}$ values are almost always well below 2 , far from the typical exclusion threshold of 4

In the case of additional assumptions, these are mentioned in the according sections. The selection of 12 satellites is based on a conservative, reasonable lower bound on the number of dual frequency corrections provided in a future GAST $\mathrm{F}$ system based on Stanisak et al. ${ }^{1}$ Stanisak et al give a maximum of 14 satellites to be provided with corrections. Nevertheless, additional information might be required in the transmission, limiting the number further. As we could show in Gerbeth et $\mathrm{al}^{12}$ that the selection algorithm performs better, the closer the number of selected satellites approaches the number of available satellites, we assessed the more demanding, conservative case.

\section{1 | Service volume and convergence time considerations}

We start with presenting results for service volume and convergence time considerations. Figure 4 shows resulting protection levels over a full day of simulation in medium latitudes, assuming $5 \mathrm{~km}$ distance to the GBAS reference point. Starting with an unconstrained optimization, ie, the best set is selected in each time step independently, different constraints are added. To get a better view of the time-behavior, we show one exemplary hour of the plot magnified in Figure 5. Figure 6 completes the picture with according histograms.

The first constraint we take a look at is limiting the exchange rate to match with the airborne filter convergence times. As we assume 100-second processing modes here, an exchange can only occur every 100 seconds. In general, this has a minor influence, as sets change rather slowly over time in general. When considering 30 -second processing modes, the influence becomes basically negligible. 


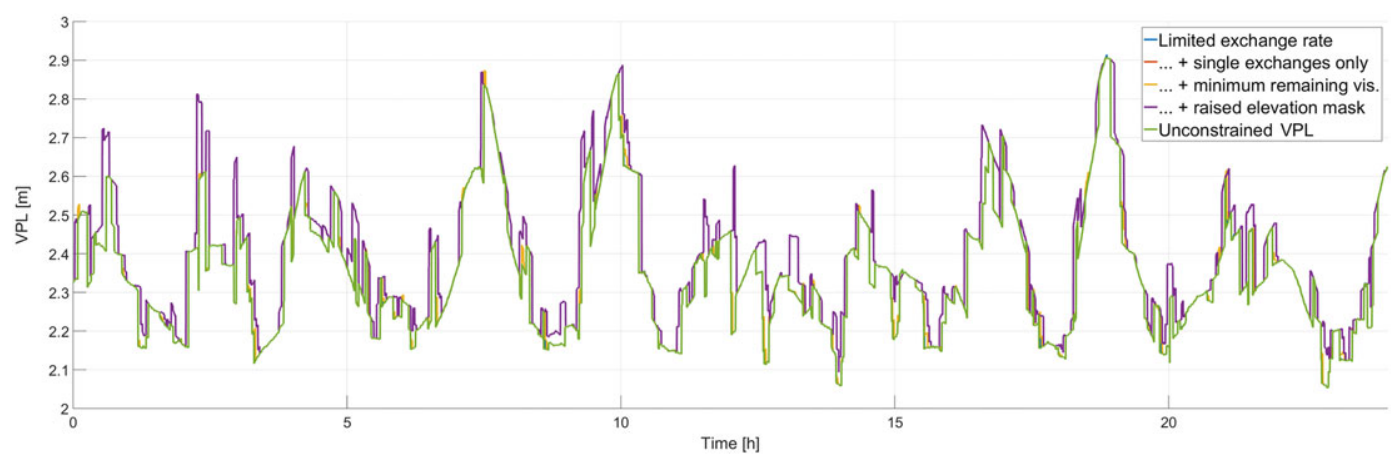

FIGURE 4 Influence of different constraints on achieved nominal protection levels over 1 day [Color figure can be viewed at wileyonlinelibrary.com and www.ion.org]

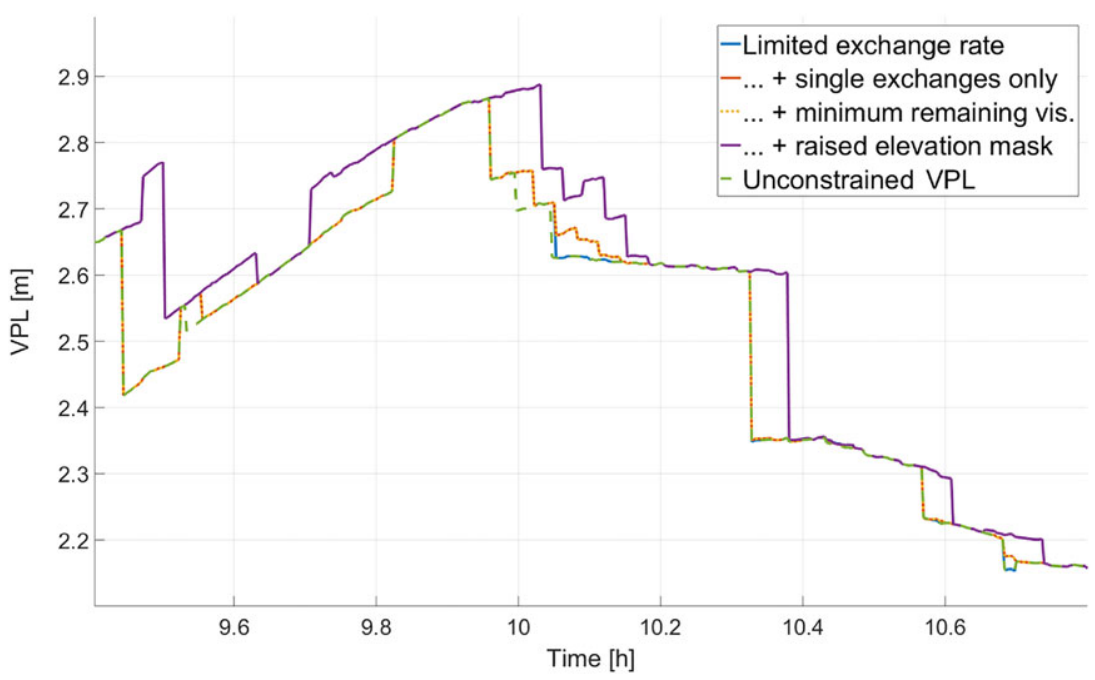

FIGURE 5 Zoom of Figure 4 to make time behavior visible [Color figure can be viewed at wileyonlinelibrary.com and www.ion.org]
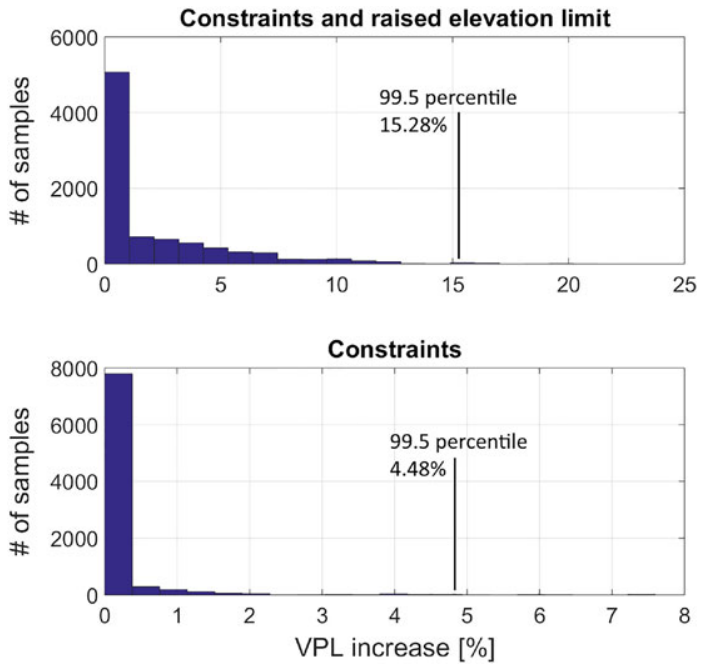

FIGURE 6 Histogram of VPL increases due to selection constraints. Bottom histogram includes the exchange constraints, the top one additionally a raised elevation mask [Color figure can be viewed at wileyonlinelibrary.com and www.ion.org]
Secondly, we limit each of the set modifications to a single satellite and ignore satellites that are setting below the elevation mask within the convergence time of $100 \mathrm{sec}-$ onds. During the simulated day, on about 100 occasions, more than one satellite was exchanged in comparison to the set from the time step before. In these cases, the selection comes out of track and needs some time to "converge" back to the optimum. This can be seen in a prominent example around 10.1 hours into the simulation where actually 3 satellites change in the best set. Nevertheless, the error made here stays minor with about $5 \mathrm{~cm}$ or $2 \%$. In total, the errors are mostly negligible and last about 5 minutes at most. The exclusion of setting satellites affects the selection only in two occasions on a millimeter level. In general, it can be said that satellites in the right azimuth region make their way into the best set earlier than within the last minutes of visibility which renders this restriction unproblematic.

As a last constraint, we limit the selection to satellites whose elevation is high enough to be usable all over the service volume. We went with this simple approach of 
assuring the satellite set performance all over the area, as the more complex optimization using satellite visibility regions showed no major benefit without specific areas within the terminal area for which to optimize. This can also be seen in a comparative histogram in Figure 7 . Especially from Figure 4, it becomes clear that the raised limit has the biggest influence on the VPL, occasionally causing some significant spikes. A statistical overview on the 24 hours is given as a histogram in Figure 6. We show the relative increase of the protection levels compared to the unconstrained VPL. While the increase in the case of the exchange constraints stays well below $10 \%$, it reaches up to $20 \%$ when introducing a raised elevation mask. Nevertheless, the influence on the performance hardly implies any consequences on the availability. The spikes primarily happen when the VPL is comparably low, and the overall worst VPL of the day is basically unchanged regardless of the constraints.

In Figure 7, we tie in with the previous histograms and show the variability over latitudes. From knowledge of the satellite orbits, it is expected that the raised elevation mask has a growing influence when more low elevation satellites are present. This matches with the results in the three histograms: We see the distribution spreading out when going further towards the pole. Errors increase up the $34 \%$ in the case of a station at $75^{\circ}$ North. However, even in high latitudes with unfavorable satellite geometries, the general performance shows to be comparable with the "easier" scenarios close the the equator.

We additionally plot the results when using the second described approach of using satellite visibility regions to optimize the ground selection. While this shows some ben-
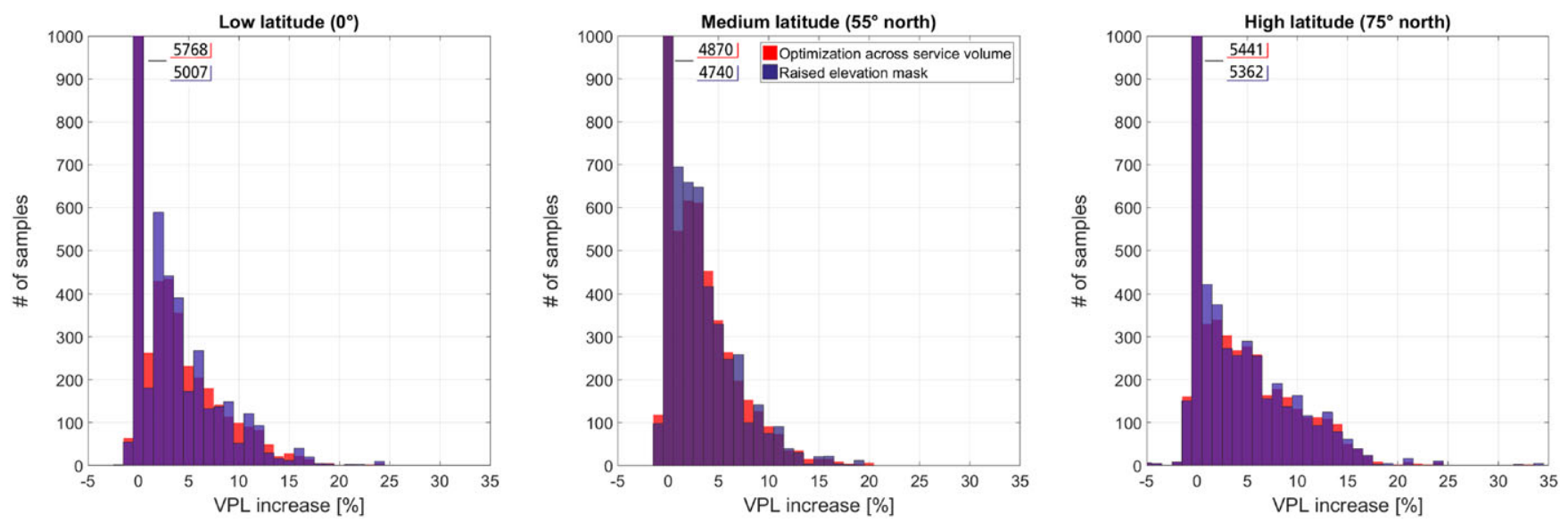

FIGURE 7 Influence of the constraints in the selection on the average VPL performance for different latitudes. Center histogram compares simple raised elevation mask with "exhaustive" optimization over service volume [Color figure can be viewed at wileyonlinelibrary.com and www.ion.org]

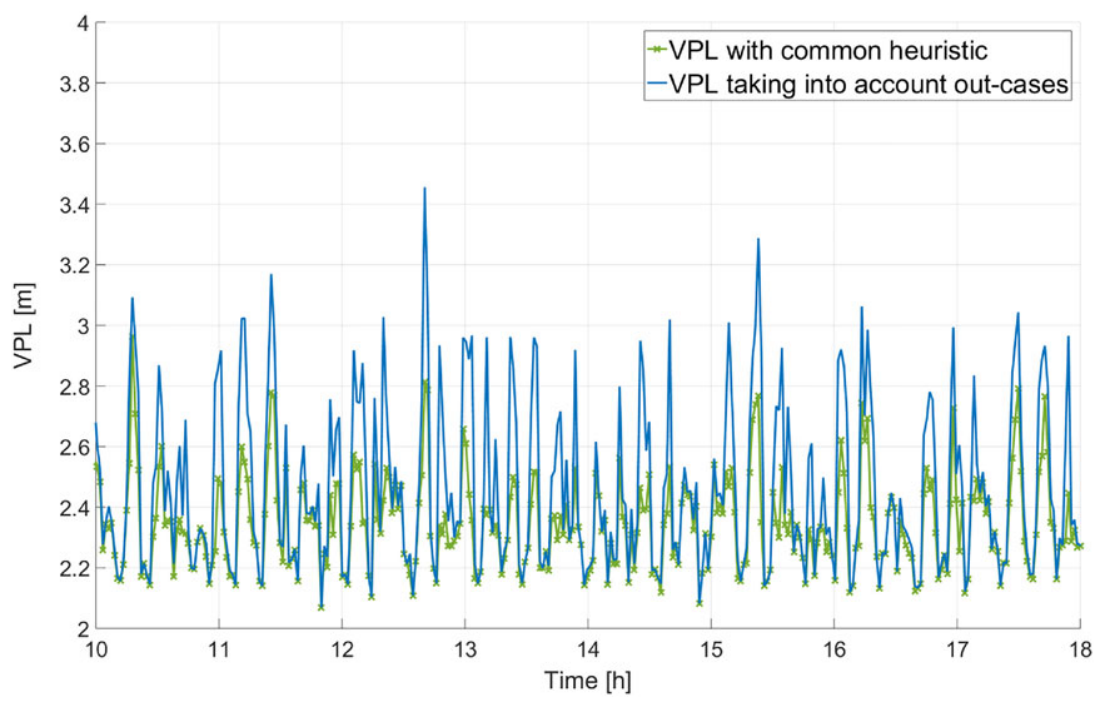

FIGURE 8 VPL over time, comparing common heuristic and optimization for satellite out cases. In this simulation, no outages are present [Color figure can be viewed at wileyonlinelibrary.com and www.ion.org] 
efit in special cases with only a limited area of the service volume actually used by approach routes, the performance when no such side conditions are given is comparable to the straightforward rise of the elevations mask.

\section{2 | Consideration of satellite-out cases}

In the second part of this subsection, we will study the VPL performance implications when considering satellite-out cases during the selection process. We limit the shown plots to a location in medium latitudes here, as the results were fairly similar for all locations. Figures 8 and 9 show the achieved protection levels for a part of the simulated time as well as the according histogram when no outages occur. In terms of the adjusted selection, this can be

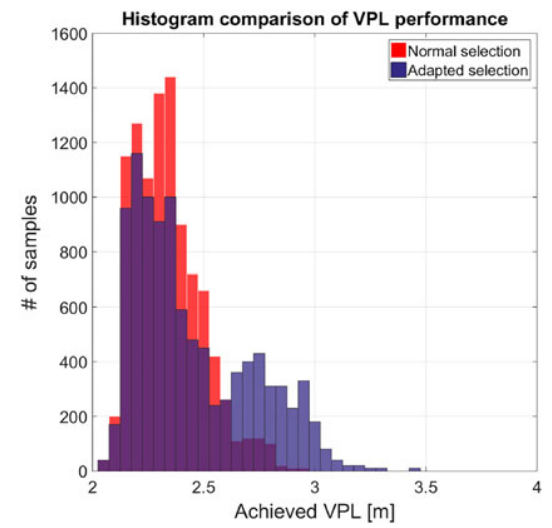

FIGURE 9 Histogram for to the full simulated day in Figure 8 [Color figure can be viewed at wileyonlinelibrary.com and www.ion.org] seen as "worst case," as there is no gain possible. Consequently, the achieved protection levels are mostly larger compared to the common selection. The average VPL over the day increases by about $5 \%$ in this case, with a maximum increase of $25 \%$.

This expected negative influence starts to be compensated by the more robust sets when we start taking out satellites. Between one and two affected satellites, the mean performance evens out, and for three satellites, we have a slightly better average performance as seen in Figures 10 and 11. In this case, we take out three random satellites (according to the probabilities from Equation 5) in every simulated epoch. Even more relevant than the average performance is that spikes, as they start to occur in the commonly selected sets, are less likely and less

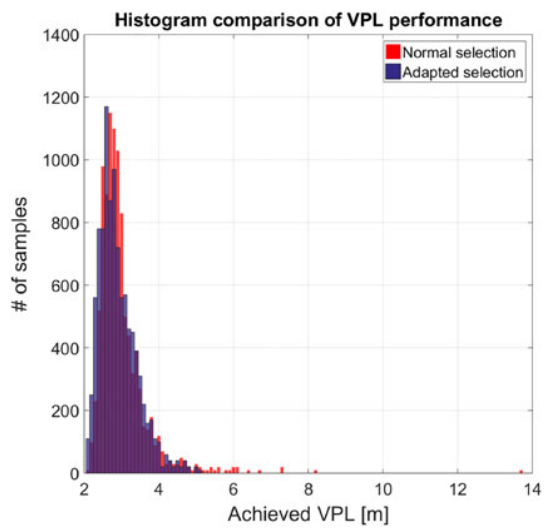

FIGURE 11 Histogram for the full simulated day in Figure 10 [Color figure can be viewed at wileyonlinelibrary.com and www.ion.org]

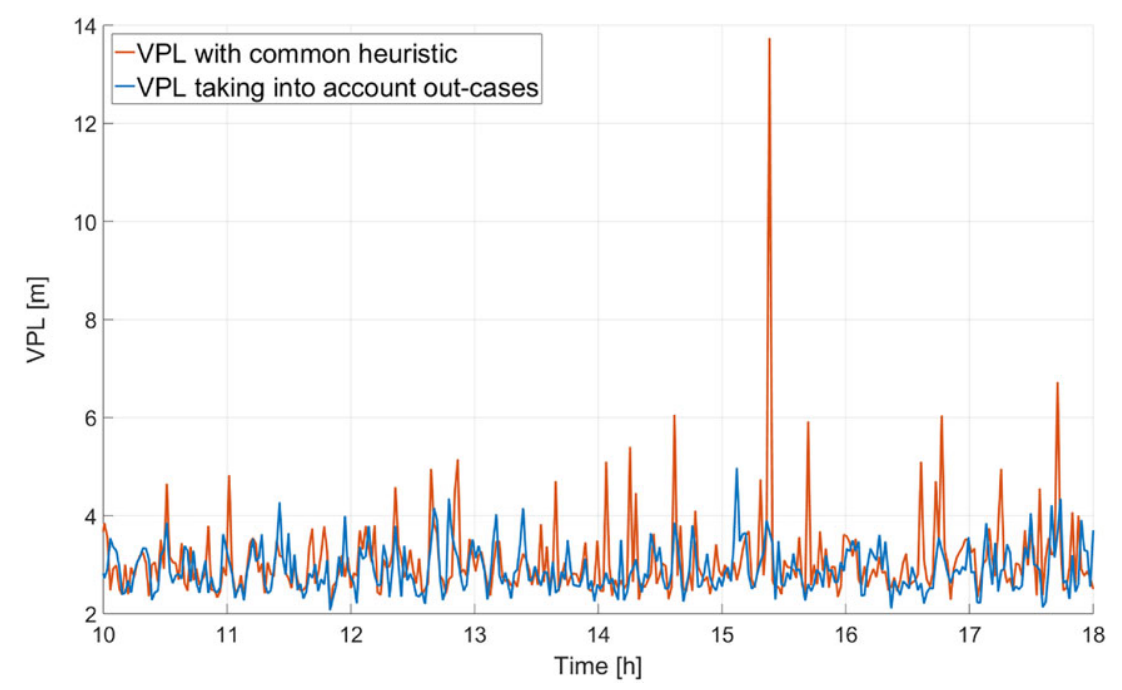

FIGURE 10 VPL over time, comparing common heuristic and optimization for satellite out cases. In this simulation, three random outages are present [Color figure can be viewed at wileyonlinelibrary.com and www.ion.org] 


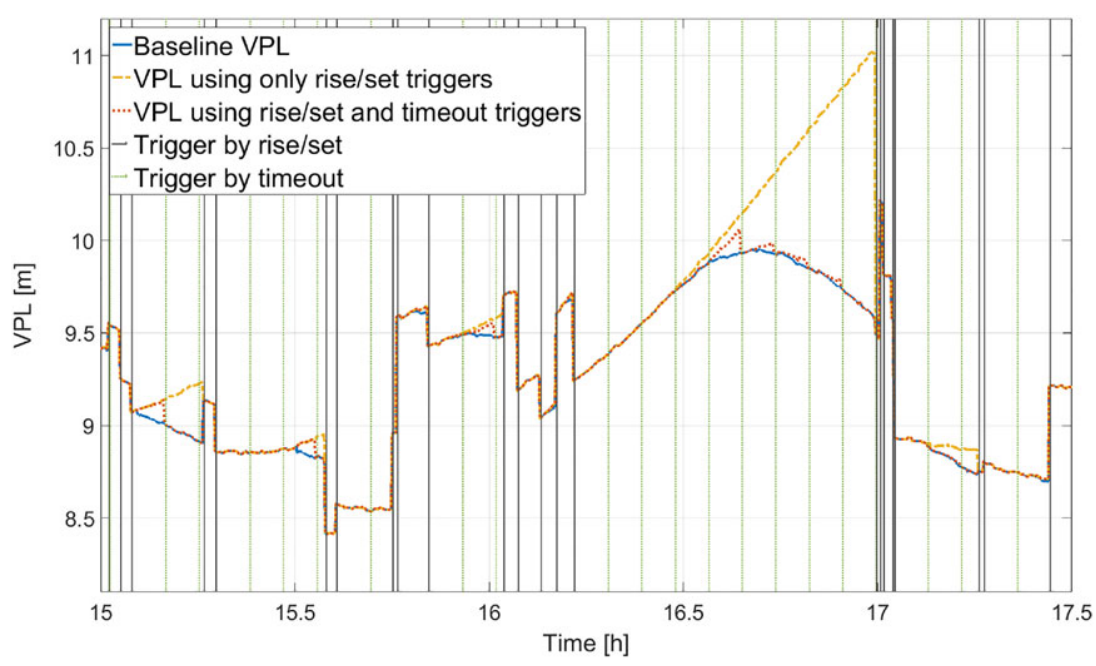

FIGURE 12 Snapshot of period with most significant divergence within 24-hour simulation time. Baseline VPL (SBAS in this case) is optimized at each epoch. In the other cases, the selected sets are kept except for trigger events [Color figure can be viewed at wileyonlinelibrary.com and www.ion.org]

pronounced with the modified selection strategy. Giving up some nominal performance can help even in some of the simulated cases to keep the system available while a commonly selected set of 12 satellites would be rendered unusable by the three lost satellites. Even though it might be unlikely, it is definitely not impossible to lose 3 out of 12 selected satellites particularly when thinking of $180^{\circ}$ turns within the terminal area as seen from the typical approach routes of Frankfurt in Figure 2. Taking this into account-maybe even with an azimuth dependent outage probability based on the approaches-could therefore help increase the availability, considering the average performance leaves some margin.

\section{3 | Airborne selection strategies}

After the ground related results in the last two subsections, we turn toward the airborne side now. As in GBAS, the airborne side would not perform any selection on its own but would rely on the provided corrections (and therefore selection) from the ground. We perform simulations for an SBAS scenario here. In Figure 12, we show a part of a 24-hour simulation of stand-alone airborne selection (12 satellites from dual constellation). For the baseline VPL, the selection is performed in every epoch to get the best performance with highest computational effort. In the red dotted line, we see the achieved VPL when we limit the number of set searches drastically by only performing them when a satellite sets or rises. While the red dotted curve follows the baseline quite well for major parts of the day, between hour 16 and 17 in a longer segment without triggers, the VPL starts to drift away significantly.
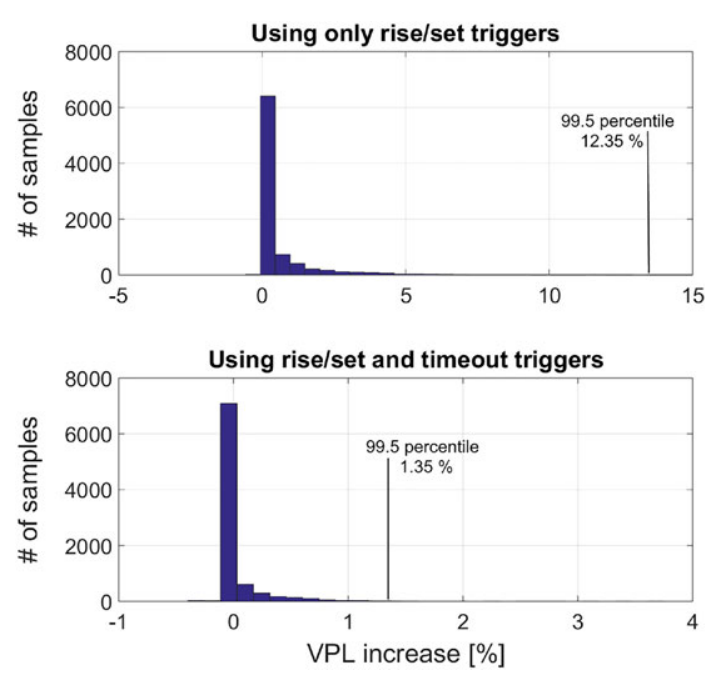

FIGURE 13 Histogram of relative SBAS VPL increases within 24 hours of simulation. Top plot uses only satellites sets and rises. Bottom includes trigger after fixed timeout [Color figure can be viewed at wileyonlinelibrary.com and www.ion.org]

When we add a simple timed trigger, in this case every 5 minutes, we can see the VPL following the baseline very well. A simple trade-off is present here between the wait interval until a new set is searched and the achieved performance. Nevertheless, with a very rare trigger as in the example, we already achieve very good results. Looking at the histograms in Figure 13, we see that the VPL increases by less than $1 \%$ most of the times for the latter case. For most applications, especially thinking of computationally limited UAS applications, this would probably be enough while reducing the computational cost significantly. 


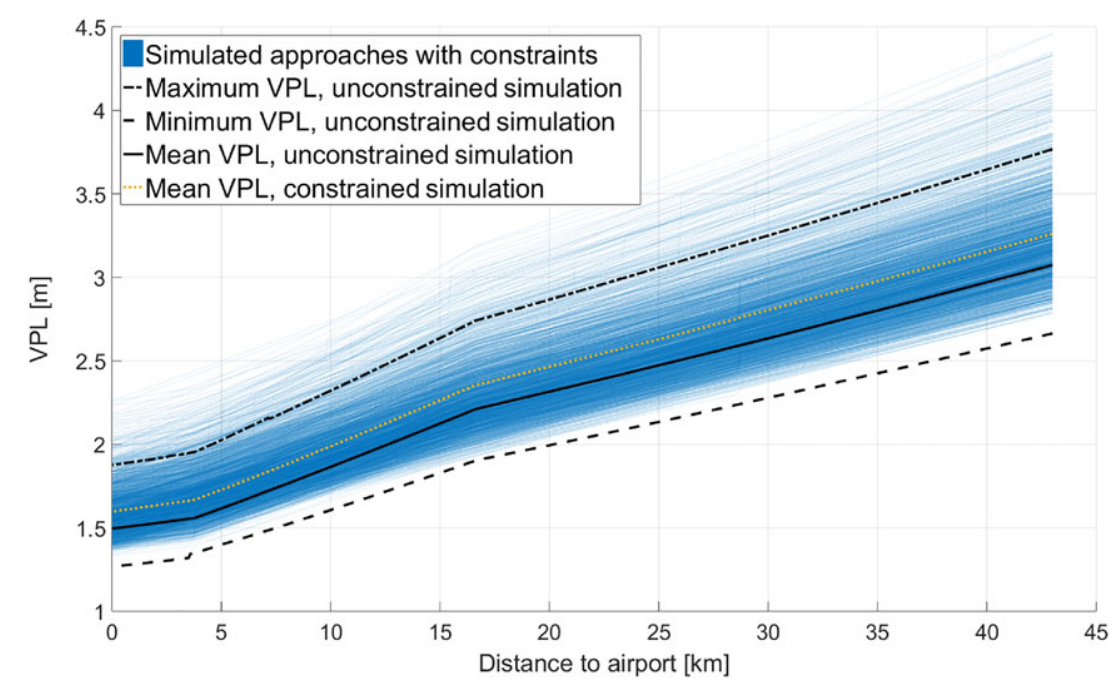

FIGURE 14 Stacked VPLs from 1000 simulated approaches combining all the previous constraints. As comparison, we show minimum, maximum, and mean VPL along the approach for the unconstrained case [Color figure can be viewed at wileyonlinelibrary.com and www.ion.org]

In terms of the proposed satellite exchange scheme, we do not show explicit results in this part, but utilize the algorithm in the conclusive simulation described in the following.

\section{4 $\mid$ Bringing the pieces together}

In a final plot, we want to bring all the previous bits and pieces together and show the VPL performance during 1000 simulated landings toward an airport in medium latitudes. All simulations start at a distance of $43 \mathrm{~km}$ from the ground reference station, ie, at the edge of the service volume. Start times of the simulations are equally spaced within 10 days to get diversity in terms of satellite geometry. The aircraft starts with 12 tracked satellites optimized for stand-alone positioning. Therefore, an alignment might be necessary in the first minutes of the approach.

The ground station incorporates all the previous constraints: limited exchange rate and exchange timeout, raised elevation mask for optimization across the service volume and consideration of out-cases. Figure 14 shows the achieved protection levels as a cloud of plots (thin semi-transparant lines) as well as the achieved mean at each distance (yellow dotted line). Additionally, the minimum, maximum, and mean values of the same approaches, not taking into account any of the constraints, are given for comparison.

We can find again in the plots the impact on the performance that could be expected from the previous simulations. The average VPL increased by about $6 \%$ to $8 \%$ along the approach, both following the same shape (introduced by speed and height profile, see Gerbeth et $\left.\mathrm{al}^{16}\right)$, as expected. In terms of the maximum VPLs, we see some rare outliers which exceed the unconstrained maximum by about $18 \%$ to $21 \%$. This is also understandable, as we already saw some significant outliers in Figure 6 when introducing the raised elevation limit. Some geometries are especially susceptible to a loss of low elevation satellites, for example, when the only low satellite in a wider azimuth range is missing. However, the VPLs during all the approaches in the simulation stay well below the vertical alert limit which is $10 \mathrm{~m}$ close to the airport and increases towards the edge of the service volume.

\section{4 | CONCLUSIONS AND FUTURE WORK}

In this paper, we studied various operational constraints and presented possible modifications of satellite selection in GBAS. First, we investigated the implications of different satellite visibilities and filter convergence times on the ground station selection. We showed that taking these constraints into account has visible but not significant influence on the expected performance for airborne users.

We showed in a general example that it is possible to trade off nominal performance versus more tolerance against satellite outages, mostly due to banking of the plane. In a real application, this can be further adapted to each specific airport. As the most common approach routes are known, it is possible to design an azimuth-elevation-dependent map of the probability of losing track of a satellite. This allows optimizing performance and will be part of further studies. All these 
constraints are helping to avoid situations where an unfavorable combination of tracked satellites in the air and corrections from the ground cause airborne users to lose the GBAS service during approach, which in the end improves availability of the according station itself.

Furthermore, we showed how simple triggers within the selection can be used to reduce the computational demand on airborne users significantly while retaining most of the performance and even allowing a flexible trade-off. Additionally, we proposed a straightforward scheme for satellite exchanges when transitioning from stand-alone GNSS navigation into GBAS.

Finally, we combined all previous studies and showed the effect on the performance during approaches when all of the constraints are taken into account. Results with protection levels well below the alert limit and average increases below $10 \%$ show that even when considering many constraints and limitations, satellite selection in a multi-constellation GBAS stays applicable.

\section{ORCID}

Daniel Gerbeth (D) https://orcid.org/0000-0002-7495-7361 Michael Felux (D) https://orcid.org/0000-0002-8368-4422

\section{REFERENCES}

1. Stanisak M, Lipp A, Feuerle T. Possible VDB formatting for multiconstellation/multi-frequency GBAS. Proceedings of the $28^{\text {th }}$ International Technical Meeting of the Satellite Division of The Institute of Navigation (ION GNSS+ 2015); September 2015; Tampa, FL:1507-1518.

2. Zhang $M$, Zhang J. A fast satellite selection algorithm: beyond four satellites. IEEE J Sel Top Signal Process. October 2009;3(5):740-747.

3. Song D, Zhang PF, Fan GC, Xu CD. An algorithm of selecting more than four satellites from GNSS. International Conference on Advanced Computer Science and Electronics Information (ICACSEI 2013); 2013; Beijing, China. https://doi.org/10.2991/ icacsei.2013.33

4. Park CW, How JP. Method and apparatus for selecting optimal satellites in global positioning system. US Patent 6,727,850 B2; 2004.

5. Kihara M, Okada T. A satellite selection method and accuracy for the global positioning system. NAVIGATION. 1984;31:8-20.

6. Blanco-Delgado N, Nunes FD. Satellite selection method for multi-constellation GNSS using convex geometry. IEEE Trans Veh Technol. November 2010;59(9):4289-4297.
7. Blanco-Delgado N, Nunes FD, Seco-Granados G. Relation between GDOP and the geometry of the satellite constellation. International Conference on Localization and GNSS (ICL-GNSS); 2011; Tampere, Finland:175-180.

8. Phatak MS. Recursive method for optimum GPS satellite selection. IEEE Trans Aerosp Electron Syst. 2001;37(2):751-754.

9. Song J, Xue G, Kang Y. A novel method for optimum global positioning system satellite selection based on a modified genetic algorithm. PLOS ONE. Y. Wang Ed. March 2016;11(3):e0150005.

10. Simon D, El-Sherief H. Navigation satellite selection using neural networks. Neurocomputing. 1995;7(3):247-258.

11. Walter T, Blanch J, Kropp V. Satellite selection for multi-constellation SBAS. Proceedings of the 29th International Technical Meeting of the Satellite Division of The Institute of Navigation (ION GNSS+ 2016); September 2016; Portland, OR:1350-1359.

12. Gerbeth D, Felux M, Circiu M-C, Caamano M. Optimized selection of satellite subsets for a multi-constellation GBAS. Proceedings of the 2016 International Technical Meeting of The Institute of Navigation; January 2016; Monterey, CA:360-367.

13. Swaszek PF, Hartnett RJ, Seals KC, Swaszek RMA. A temporal algorithm for satellite subset selection in multiconstellation GNSS. Proceedings of the 2017 International Technical Meeting of The Institute of Navigation; January 2017; Monterey, CA:1147-1159.

14. Gerbeth D, Martini I, Rippl M. Satellite selection methodology for horizontal navigation and integrity algorithms. Proceedings of the 29th International Technical Meeting of the Satellite Division of The Institute of Navigation (ION GNSS+ 2016); September 2016; Portland, OR:2789-2798.

15. RTCA DO-253D. Minimum operational performance standards for GPS local area augmentation system airborne equipment. Technical Report 253D, Radio Technical Commission for Aeronautics; 2017.

16. Gerbeth D, Circiu M-S, Caamano M, Felux M. Nominal performance of future dual frequency dual constellation GBAS. International Journal of Aerospace Engineering. 2016;2016:1-20.

17. Kumar R, Battista G, Osechas O. Demand-based placement of LDACS ground stations to achieve RNP 0.3 accuracy for APNT. Proceedings of the 2017 International Technical Meeting of The Institute of Navigation; January 2017; Monterey, CA:1227-1241.

How to cite this article: Gerbeth $\mathrm{D}$, Caamano M, Circiu M-S, Felux M. Satellite selection in the context of an operational GBAS. NAVIGATION. 2019;66:227-238. https://doi.org/10.1002/navi.284 